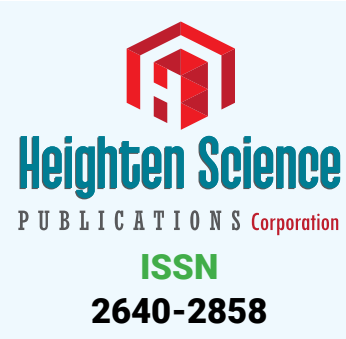

*Address for Correspondence: Dr. Vesna Ambarkova, PhD,.MSc, DDS, University St. Cyril and Methodius, Faculty of Dental Medicine, Department of Paediatric and Preventive Dentistry, Mother Theresa 17 University Dental Clinic Center Sv.Pantelejmon Skopje 1000, Republic of Macedonia, Tel ++38970686333 Email: vesna.ambarkova@gmail.com

Submitted: 24 May 2017

Approved: 06 June 2017

Published: 08 June 2017

Copyright: @ 2017 Vesna A. This is an open access article distributed under the Creative Commons Attribution License, which permits unrestricted use, distribution, and reproduction in any medium, provided the original work is properly cited

Check for updates

\title{
The Neuromuscular diseases in Pediatric Dental Office
}

\author{
Ambarkova Vesna* \\ University St. Cyril and Methodius, Faculty of Dental Medicine, Department of Paediatric and \\ Preventive Dentistry, Skopje, Republic of Macedonia
}

\section{ABSTRACT}

The neuromuscular disorders may be hereditary, autoimmune, and in some cases with unknown etiology. These diseases are characterized by progressive course, muscle weakness, and in an advanced stage with binding the patient to a wheelchair. This group includes a number of diseases, but from the dental perspective, the most interesting are muscular dystrophy, multiple sclerosis and myasthenia gravis. Neuromuscular disorders affect the oral cavity and the impact on oral hygiene procedures should be monitored with great attention.

\section{THE MUSCULAR DYSTROPHY}

Muscular dystrophy is an inherited disease of skeletal muscle which leads to progressive degenerative changes. The incidence of the muscular dystrophy is $1,4: 10.000$, usually occurs only in boys, and because of the mutant gene has a recessive inheritance by $\mathrm{X}$ chromosomes.

Children normally develop in the first year, with the difference that a little later start to sit independently. Also children have difficult to climb on a chair, which often the earliest sign and never starts is walking before 18 months ago.

The disease develops gradually and rapidly, so that by the end of the first decade, the muscle dystrophy is so expressed that binding of the child to a wheelchair is imminent. At the end of the second decade, the propensity to recurrent infections of the airways is much expressed.

Dystrophic changes very early affect heart muscles leading to premature death. Survival to twenty years of age occurs very rarely. About twenty-five percent of all patients have a problems with memory. Unfortunately, there is still no effective treatment, nor medical professional can stop progression of the disease. Social assistance to the family of the affected child is the only thing that can be done to help the child.

\section{DENTAL TREATMENT}

The regular visits to the dentist from the early age are needed, because with the application of preventive and prophylactic measures we can maintain oral health. Limited opening of the mouth may occur in later stages of the disease, because of the muscle contraction and then will be nearly impossible to do any dental treatment. Dental therapeutic treatment is implemented by local anesthesia. The application of general anesthesia is contraindicated, because of sluggish movements of the chest muscles, and additional risk represents cardiac arrest or the appearance of malignant hyperpyrexia. 
Bezak et al. in their case series describe patients with Congenital Myopathies (CMs) or Congenital Muscular Dystrophies (CMDs), which very often have dentofacial malocclusions that induced functional problems with feeding and drooling and also psychosocial problems. Orthognathic surgery can be judiciously considered in these patients, which require multidisciplinary pre- and postoperative evaluation and care over lengthy hospital stays with a high risk of respiratory complications [1].

Matsumoto et al. used auricular cartilage graft to elevate the lip in patient with facioscapulohumeral muscular dystrophy. This procedure is less invasive and with a longer-lasting effect. Authors believe that this is an appropriate technique to correct similar patients of lip deformity [2].

Van der Engel-Hoek et al. in their study talk about dysphagia in Duchenne muscular dysphagia (DMS) and its worsens with age. Also he describe mastication problems in consecutive stages and determine related pathophysiological aspects of masticatory muscle structure, tongue thickness, bite force and dental characteristics [3].

\section{Multiple sclerosis}

Multiple sclerosis is a chronic degenerative disease of the central nervous system that can affect myelin, the white brain and spinal cord. The disease is of unknown etiology, and usually occurs suddenly in completely healthy people aged between 2040 years of age, more common in females. The appearance of demineralization of the myelin sheath of the nerves, cause numerous motor and sensory disorders in patients suffering from multiple sclerosis. Three signs characterize multiple sclerosis. They are nystagmus ("dancing eyes"), tremors and intermittent speech. Initial symptoms of the disease may occur in the form of dizziness, loss of vision, diplopia and ataxia. Intellectual ability is preserved, but in later stages of the disease may develop dementia.

The diagnosis is usually difficult because of the diversity of symptomatology. The condition is characterized by repeat attacks of acute and occurrence of multiple lesions. Some can be only one attack after coming to a complete return to normal state, while in others there is only few exacerbations with asymptomatic periods, but does not come to a complete return to normal condition, in fact disease has a progressive course and with binding the patient to a wheelchair. Treatment of the multiple sclerosis is symptomatic. Giving of corticosteroids, prednisone, $60 \mathrm{mg}$ per day for 5 days, may be useful in acute exacerbations.

Dental treatment. During a dental intervention, the patient have to be protect with antibiotic therapy because infection can occur. Dental examination and treatment must be short, because of the pronounced weakness of the muscles and the inability of the patient to hold open the mouth longer.

\section{Cerebral palsy}

Cerebral palsy represents a non-progressive lesion of the brain, which is characterized by the inability to coordinate the movements, as well as the emergence of involuntary movements. Presents one of the common form of physical disability. The incidence of the appearance ranges from 1, 5-3: 1000, but have often appears within boys.

Cerebral palsy is not a specific disease, but rather a set of symptoms that are caused by insult and permanently damaging of the brain which can occur prenatally (viral infection of the pregnant women), in the perinatal period (due to cerebral anoxia at the birth of baby), or postnatally (due to the meningitis, trauma of the head of incompatibility or rhesus factor). The children suffering from cerebral palsy is often mentally retarded (60\%), have a disturbance in the speech $(70 \%)$, have visual disability (25\%) and hearing disorder (5\%), epilepsy (30-50\%) and contractures in 
the joints. Their speech is damaged due to unnatural movements of the language and loud wire, thus giving a false picture of the mental retardation.

There are different degrees and symptoms of the disease. In some cases the symptoms are minimal, whereas in the other such a large degree of involvement exist, due to which it is necessary patients to use wheelchair, lifelong personal help in all activities, and even more in most difficult cases hospitalization of the patient.

Cerebral palsy may be accompanied by involuntary movements of the body, epilepsy, a disturbance in the holding of hygiene, dysfunction of the sensory functions and insufficient mental development level.

There are four types of cerebral palsy: spastic, athetoid, ataxic and combined type. Spastic type is characterized with sturdy and rigid muscles, also sometimes the muscles of the lips, language and throat are affected, epilepsy, dysarthria and insufficient mental development level. Athetoid cerebral palsy is characterized with hypotonia, slow, uncontrolled movements, frequent changes in muscles tone, dysarthria, ataxic cerebral palsy, the problem of the holding the balance and problem with the perception of the object distance, unsafe walking, hypotonia and tremor.

\section{Combined type which manifests itself with different symptoms}

As far as the general principles on taking care includes necessity to provide free space for movement of the patient, to snap the equipment and instruments outside the reach of patients.

The specifics that are characteristic of the oral health condition of people suffering from cerebral palsy include the low level of oral hygiene and high distribution on periodontal illness, high percentage on untreated dental caries, orthodontic anomalies, dysphagia, bruxism, drooling, the urge to vomit and trauma in orofacial region. The maintenance of the oral hygiene represents an important part of the general and the individual preventive program within the people suffering from cerebral palsy.

The periodontal illness is usual condition within the persons suffering from cerebral palsy as a consequence of the bad oral hygiene due to focus to the treatment of the primary illness. Difficulties in the maintaining of oral hygiene are due to physical inability to take an action of brushing teeth, and possible orthodontic problems. An additional factor represents favoring gingival hyperplasia caused by medicaments that are used in the therapy of the primary illness.

\section{Myasthenia gravis (Erb-Goldflam-ova disease)}

Myasthenia gravis is neuromuscular, autoimmune disease characterized by weakness of the muscles due to interruption of neuromuscular transmission. Actually, acetylcholine receptors on the terminations of the nerves of skeletal muscle is blocked by antibodies, and cannot respond to the liberated acetylcholine at the ends of the nerves endings of muscles.

The incidence of the myasthenia gravis is $1: 20.000$, and somewhat more common among females (3:2). The first manifestations of the disease are within muscles of the face and eyes, and in the later stage of the disease, muscles of the larynx, pharyngeal and respiratory muscles are affected. The characteristic signs of the disease, manifest themselves in the form of difficulties in the process of mastication and swallowing at the end of the meal, ptosis of the eyelid in the afternoon and especially evening hours, double vision, slurred speech, weakened voice in a long speech, inability to close the mouth, weakness of the leg muscles after walking, dyspnea and hyperventilation. In a large percentage of patients with the progression of the disease, comes to increasing of the thymus. 
Treatment begins by giving anticholinesterases to increase neuromuscular transmission. If it does not give satisfactory results, further treatment include corticosteroids and thymectomy. The implementation of plasmapheresis, before making thymectomy significantly helps in $30-45 \%$ of patients. Anti-acetylcholinesterase agents (e.g., Mestinon $\AA$ ) allow acetylcholine to remain at the neuromuscular junction longer than usual so that more receptor sites can be activated. Treatment goals are individualized. Corticosteroids (e.g., prednisone) and immunosuppressant agents (e.g., Imuran ${ }^{\circledR}$ ) may be used to suppress the abnormal action of the immune system that occurs in MG. Thymectomy (surgical removal of the thymus gland) is another treatment used in some patients. The thymus gland lies behind the breastbone and is an important part of the immune system. When there is a tumor of the thymus gland (in $10-15 \%$ of patients with $\mathrm{MG}$ ), it is always removed because of the risk of malignancy. The degree to which the thymectomy helps varies with each patient.

Fourteen drugs were identified by Cockburn et al. [4], for the treatment of MS progression and 13 drugs for the treatment of MS symptoms. For these medications, 18 oral side effects were documented: xerostomia was the most common, followed by dysgeusia, dysphagia, mouth ulceration and sinusitis. Anticholinergic drugs caused xerostomia while immunosuppressants resulted in more infection related side-effects. Application of digital technology in the treatment of a patient with myasthenia gravis is presented by AlHelal et al. [5]. In his case report, a 74 year old woman with excessively resorbed mandibular residual alveolar ridge came to Loma Linda University School of Dentistry for treatment.

\section{Dental treatments}

Dental interventions must be of short duration, because of rapid tiredness that patients experience after long holding the mouth open. Patients in dental chair felt much more comfortable sitting in terms of supine position. It should be borne in mind that patients who take anticholinesterase present increased salivation. During giving of local anesthesia, dentists should use lidocaine and anesthetics pelated to lidocaine, because the application of procaine is not desirable. The application of general anesthesia for dental care in patients with myasthenia gravis should be avoided, due to weakness of the respiratory muscles.

Dental management of patients diagnosed with Myasthenia gravis (MG) presents a challenge to the oral health care provider. Patil et al. in his article review the etiology, pathogenesis, diagnosis, and clinical signs and symptoms associated with MG, highlighting the role of the oral health care provider in the process of diagnosis and management of the oral and dental complications that might be associated with the disease, while avoiding myasthenic crisis [6].

\section{REFERENCES}

1. Bezak BJ, Arce KA, Jacob A, Van Ess J. Orthognathic surgery in patients with congenital myopathies and congenital muscular dystrophies: case series and review of the literature. J Oral Maxillofac Surg. 2016; 74: 601-609. Ref.: https://goo.gl/0dfmjg

2. Matsumoto $M$, Onoda $S$, Uehara $H$, Miura $Y$, Katayama $Y$, et al. Correction of the lower lip with a cartilage graft and lip resection in patients with facioscapulohumeral muscular dystrophy. $J$ Craniofac Surg. 2016; 27: 1427-1429. Ref.: https://goo.gl/YptTKy

3. Van der Engel-Hoek L, de Groot IJ, Sie LT, van Bruggen HW, et al. Dystrophic changes in masticary muscles related chewing problems and malocclusions in Duchenne muscular dystrophy. Neuromuscul Disord. 2016; 26: 354-360. Ref.: https://goo.gl/pwtYeh

4. Cockburn N, Pateman K, Taing MW, Pradhan A, Ford PJ. Managing the oral side effects of medications used to treat multiple sclerosis. Aust Dent J. 2017; 9. Ref.: https://goo.gl/2Lgrdr

5. AlHelal A, Jekki R, Richardson PM, Kattadiyil MT. Application of digital technology in the prosthodontic management of a patient with myasthenia gravis: a clinical report. J Prosthet Dent. 2016; 115: 531 536. Ref.: https://goo.gl/GrdNV9 
6. Patil PM, Singh G, Patil SP. Dentistry and the myasthenia gravis patient: a review of the current state of the art. Oral Surg Oral Med Oral Pathol Oral Radiol. 2012; 114: 1-8. Ref.: https://goo.gl/qQyhjZ

7. Ferreira B, Da Silva GP, Gonçalves CR, Arnoni VW, Siéssere S, et al. Stomatognathic function in duchenne muscular dystrophy: A case-control study. Dev Med Child Neurol. 2016; 58: 516-521. Ref.: https://goo.gl/cW0ph3

8. van Bruggen $\mathrm{HW}$, van den Engel-Hoek $\mathrm{L}$, Steenks $\mathrm{MH}$, van der Bilt A, Bronkhorst EM, et al. Fighting against disuse of the masticatory system in duchenne muscular dystrophy: a pilot study using chewing gum. J Child Neurol. 2015; 30: 1625-1632. Ref.: https://goo.gl/464Pyd

9. Aristizabal JF, Smit RM. Orthodontic treatment in a patient with unilateral open-bite and Becker muscular dystrophy. A 5-year follow-up. Dental Press J Orthod. 2014; 19: 37-45. Ref.: https://goo.gl/9kpVh7

10. LaDonna KA, Koopman WJ, Ray SL, Venance SL.Hard to Swallow: A phenomenological exploration of the experience of caring for individuals with myotonic dystrophy and dysphagia. J Neurosci Nurs. 2016; 48: 42-51. Ref.: https://goo.gl/VTsXoj 